\title{
Simultaneous Confocal Scanning Laser Ophthalmoscopy Combined with High-Resolution Spectral-Domain Optical Coherence Tomography: A Review
}

\author{
Verônica Castro Lima, ${ }^{1}$ Eduardo B. Rodrigues, ${ }^{1}$ Renata P. Nunes, ${ }^{1}$ Juliana F. Sallum, ${ }^{1}$ \\ Michel E. Farah, ${ }^{1}$ and Carsten H. Meyer ${ }^{2}$ \\ ${ }^{1}$ Retina Service, Department of Ophthalmology, Federal University of São Paulo, 04021-001 São Paulo, SP, Brazil \\ ${ }^{2}$ Department of Ophthalmology, University of Bonn, 53012 Bonn, Germany \\ Correspondence should be addressed to Verônica Castro Lima, vecastrolima@yahoo.com.br
}

Received 23 June 2011; Accepted 17 August 2011

Academic Editor: Fernando Penha

Copyright () 2011 Verônica Castro Lima et al. This is an open access article distributed under the Creative Commons Attribution License, which permits unrestricted use, distribution, and reproduction in any medium, provided the original work is properly cited.

\begin{abstract}
We aimed to evaluate technical aspects and the clinical relevance of a simultaneous confocal scanning laser ophthalmoscope and a high-speed, high-resolution, spectral-domain optical coherence tomography (SDOCT) device for retinal imaging. The principle of confocal scanning laser imaging provides a high resolution of retinal and choroidal vasculature with low light exposure. Enhanced contrast, details, and image sharpness are generated using confocality. The real-time SDOCT provides a new level of accuracy for assessment of the angiographic and morphological correlation. The combined system allows for simultaneous recordings of topographic and tomographic images with accurate correlation between them. Also it can provide simultaneous multimodal imaging of retinal pathologies, such as fluorescein and indocyanine green angiographies, infrared and blue reflectance (red-free) images, fundus autofluorescence images, and OCT scans (Spectralis HRA + OCT; Heidelberg Engineering, Heidelberg, Germany). The combination of various macular diagnostic tools can lead to a better understanding and improved knowledge of macular diseases.
\end{abstract}

\section{Introduction}

With the advent of novel technologies, both optical coherence tomography (OCT) and confocal scanning laser ophthalmoscopy (cSLO) have been introduced successfully into the routine clinical imaging for a wide spectrum of macular diseases. The combination of these two techniques in one instrument, which offers various subsequent advantages, including exact correlation of tomographic and topographic findings, has the potential to enhance further our understanding of disease pathogenesis, diagnosis, and patient management.

In this paper, we aimed to review the history, some of the technical aspects, and important clinical applications of a high-speed, high-resolution spectral-domain OCT (SDOCT) device which also is able to combine cSLO-based fluorescein and indocyanine green angiograms, infrared, blue reflectance ("red-free"), and fundus autofluorescence (FAF) images.

\section{History}

Based on the pioneering work of Webb et al. $[1,2]$, confocal scanning laser ophthalmoscopes (cSLOs) have been developed for clinical use. Although limited by the optical properties of the human eye, they are able to achieve high-contrast images of the posterior segment. Today most scanning laser systems record their images in real time with a fast frame rate.

Optical coherence tomography was initially reported by Huang and coworkers in 1991 [3] and has had a tremendous subsequent impact on in vivo imaging of retinal diseases. It has evolved as a noninvasive technique and allows for visualization of microstructural alterations of the retinal 


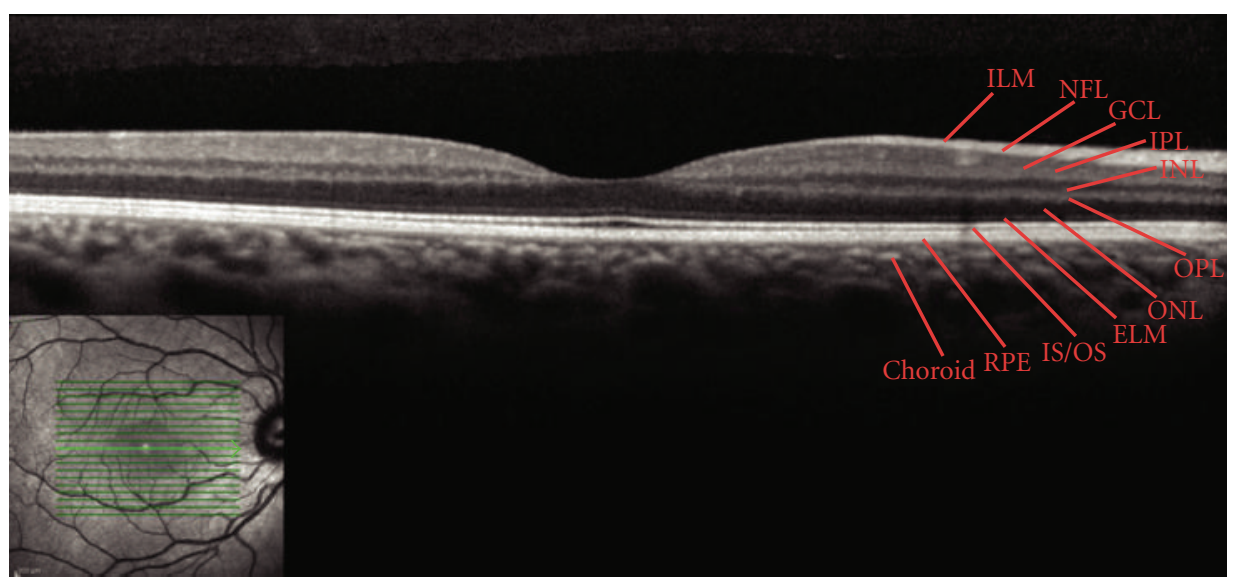

FIGURE 1: Example of a normal eye imaged with the Spectralis SDOCT. The infrared reflectance cSLO image (lower left) shows a normal fundus which corresponds to the normal SDOCT B-scan. The green lines represent the location, and the green arrow shows the exact orientation of the B-scan. All the retinal layers are indicated on the SDOCT scan (ILM: internal limiting membrane; NFL: nerve fiber layer; GCL: ganglion cell layer; IPL: inner plexiform layer; INL: inner nuclear layer; OPL: outer plexiform layer; ONL: outer nuclear layer; ELM: external limiting membrane; IS/OS: photoreceptor inner/outer segment junction; RPE: retinal pigment epithelium).

tomographic architecture. This imaging modality is now used widely by ophthalmologists for a range of indications and has become a standard diagnostic technique [4-16]. It provides images analogous to ultrasonography, but, instead of sound, it uses light waves to obtain a reflectivity profile of the tissue under investigation, measuring the time delay and magnitude of backscattered or reflected light by lowcoherence interferometry.

The OCT technique available most widely in clinical practice is referred to as time-domain OCT, because the depth information of the retina is acquired as a sequence of samples over time. This can be performed either in longitudinal cross-sections perpendicular to or in the coronal plane parallel to the retinal surface. Recently, major advances have been made regarding the image resolutionnotably the development of a high-resolution OCT-and in imaging speed, signal-to-noise ratio, and sensitivity with the introduction of an ultrahigh-resolution SDOCT [1724]. In time-domain OCT and earlier ultrahigh-resolution OCT, reference mirrors move mechanically, limiting imaging speed. In SDOCT, the reference mirror is stationary and the OCT signal is acquired either by using a spectrometer as a detector or by varying the narrowband wavelength of the light source in time (swept source). Echo time delays of light are measured by acquiring the interference spectrum of the light signal and taking its Fourier transform [25, 26]. Increasing imaging speed allows for the acquisition of images within a fraction of second, thus minimizing motion artifacts [27]. It has also become possible to acquire threedimensional volume OCT scans that achieve comprehensive retinal coverage [28].

The combination of OCT and cSLO in one instrument offers a number of subsequent advantages, including an accurate correlation of tomographic with topographic architecture of the retina, which opens new insights in the pathogenesis and morphological alterations of retinal diseases $[29,30]$. Additionally a multimodality imaging system provides a complete view of the vitreous, retina and choroid, enabling clinicians to combine information from six different modes to assess the eye: fluorescein and indocyanine green angiographies, FAF, infra-red, red-free, and SD-OCT. Simultaneous high-resolution fundus imaging and SD-OCT with the Spectralis (Heidelberg Engineering, Heidelberg, Germany) offer high-quality images with the certainty of knowing the location, leading to a significantly better diagnosis and monitoring of the patient (Figure 1).

\section{Technical Aspects}

The imaging system cSLO/OCT (Spectralis HRA+OCT) combines high speed, high-resolution SDOCT images with simultaneous recording of fluorescein and indocyaninegreen angiographies, digital infrared and blue reflectance, or FAF images. On the other hand, the Spectralis OCT has only two modes-SDOCT and infrared. Both SDOCT devices use a new proprietary eye-tracking technology, which locks onto a specific location on the retina and relocates the site at later exams to enhance the monitoring of disease progression and treatment decisions. For image clarity, the proprietary Heidelberg Noise Reduction feature takes the axial resolution from 7 microns to 3.5 microns. And the device dual-beam imaging captures a reference scan and crosssection simultaneously for reliably accurate registration.

Regarding the technical parameters of the SDOCT, 40000 A-scans are acquired per second using an optical resolution of approximately $7 \mathrm{~mm}$ in depth (axial resolution) and $14 \mathrm{~mm}$ transversally (lateral optical resolution). The centre wavelength of the OCT light source is typically between 870 and $880 \mathrm{~nm}$. OCT scans can be recorded simultaneously with fluorescein angiography, indocyanine-green angiography, FAF, infrared, and blue reflectance images. For the A-scans the scan depth is $1.8 \mathrm{~mm} / 512$ pixels, providing a digital axial resolution of $3.5 \mu \mathrm{m} /$ pixel. Spectralis scans 100 times faster than time-domain OCT and $40 \%$ faster than most other 


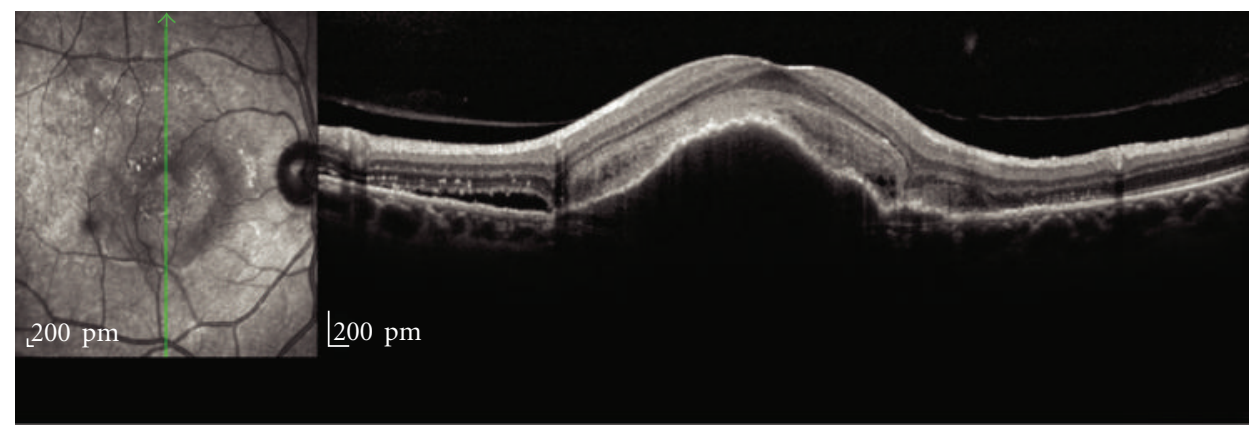

FIGURE 2: A 71-year-old man with a pigment epithelial detachment in the presence of neovascular AMD in the right eye. In the SDOCT scan the pigment epithelial detachment is readily visualized underneath the fovea associated with intraretinal cysts and subretinal fluid. It is also possible to identify the subretinal neovascular membrane and the vitreoretinal interface.

SDOCT instruments. B-scans cover a transversal range of 15,20 , or $30^{\circ}$ field of view. In the high-speed mode scan widths are 384,512 , and 768 A-scans per B-scan with a lateral digital resolution of $11 \mu \mathrm{m} /$ pixel and a scan rate of 89, 69 and 48 B-scans/second, respectively. In the highspeed mode, the vertical presentation of the OCT scan is magnified twice; therefore morphological alterations are presented disproportionately high in the vertical dimension. The accelerated imaging speed allows the acquisition of an image within a fraction of second, thus minimizing motion artefacts. Vertical and horizontal OCT scans are placed in the area of interest.

The high-resolution modes encompass a scan width of 768,1024 , and $1.536 \mathrm{~A}$-scans per B-scan with a lateral digital resolution of $5 \mu \mathrm{m} /$ pixel at a scan rate of 48,37 , and 25 $\mathrm{B}$-scans/second, respectively. High-resolution fundus images provide clear cross-sectional scans of the retinal anatomical structure, including the retinal surface, intraretinal alterations, as well as subretinal morphologic pathologies.

Sequences of B-scans can be acquired to image a full volume. These volume scans can be obtained at 15, 20 and $30^{\circ}$ field of view. The number of B-scans per volume can be adjusted from 12 to $96 \mathrm{~B}$-scans per $10^{\circ}$. In addition, it is also possible to acquire $3 \mathrm{D}$ volumetric OCT scans for comprehensive analysis of the entire retina and, therefore, for 3D mapping of pathologic alterations within the retinal layers including the RPE.

\section{Clinical Applications}

The new technology of the SDOCT has improved the visualization of intraretinal morphologic features allowing the evaluation of the integrity of each retinal layer and in vivo visualization of microstructural morphology of the retina. The high-speed, high-resolution SDOCT (Spectralis) has been applied over the last few years to investigate morphological substrates for alterations in eyes with various macular disorders.

One of the most important clinical applications of this device is to help guiding diagnosis and treatment of patients with age-related macular degeneration (AMD). In early stages of AMD, drusen can be detected at their specific location [29]. Small drusen appears as localized detachments of the retinal pigment epithelium (RPE) with intact layered architecture of the photoreceptor segments. In large drusen, RPE elevation and derangement can be seen in association with a disrupted photoreceptor band. Additionally in cases of reticular drusen, another example of early AMD, the SDOCT scan shows alterations in the outer retinal and RPE layers. These ultrastructural characteristics may allow distinguishing subclasses of drusen and may allow identifying biomarkers for disease severity or risk of progression [31]. In cases of dry AMD with geographic atrophy, SDOCT scans also can confirm loss of the RPE monolayer along with atrophy of the outer neurosensory retinal layers. In most eyes with geographic atrophy the inner retinal layers are unchanged, whereas the outer retinal layers show alterations in all eyes. SDOCT provides adequate resolution for quantifying photoreceptor loss and allows visualization of reactive changes in the RPE cells in the junctional zone of geographic atrophy $[30,32,33]$. Finally SDOCT scans can be useful to delimitate and better visualize areas of pigment epithelium detachments (PEDs) and choroidal neovascular membranes in cases of wet or exudative AMD (Figure 2). The combination of the FA and the SDOCT with high-resolution and real-time mean image elaboration may enhance the detailed visualization of activity in new choroidal neovascularization, such as presence of subretinal, intraretinal, or sub-RPE fluid, intraretinal cysts, or a combination of them $[34,35]$. The high sensitivity on neovascular activity expressed by the SDOCT features may be helpful in clinical practice, reducing the need of angiographies for treatment decisions [36].

It is well known that OCT imaging in patients with diabetic macular edema is able to reveal several structural changes in the retina, such as epiretinal membranes, intraretinal and subretinal fluid, and cystoid macular edema $[37,38]$. It is a very useful tool for diagnosis, especially in challenging cases, and treatment followup. Only a limited number of studies using different SDOCT devices for assessing diabetic macular edema and diabetic retinopathy have been published [39-42]. In cases of diabetic macular edema, SDOCT has enabled us to analyze with more details the integrity of the outer retinal layers, which includes the external limiting membrane, the photoreceptors junction, the RPE, and Bruch's membrane. One first report using the Spectralis SDOCT showed the importance of the integrity 
of the external limiting membrane and the photoreceptors junction as a prognostic feature of visual improvement after treatment for diabetic macular edema [42].

Spectral-domain OCT has dramatically improved the visualization of the vitreomacular interface and posterior hyaloid membrane and has become a very important tool for the diagnosis and followup of patients with alterations of the vitreoretinal interface. In cases of epiretinal membranes and macular pucker, vitreoretinal adhesions at the peak elevation and retinal wrinkling can be seen in the cSLO image. In the correspondent SDOCT cross-sectional image the wrinkling of the inner retinal surface and thickening of the neurosensory retina, particularly pronounced in the outer nuclear layer and the innermost neurosensory layers, can be observed [29]. Also, in cases of macular hole, besides other features that have been well described, SDOCT can demonstrate disruption of photoreceptors junction and imaging this structure is a method of assessing structural integrity of the photoreceptors before and after macular hole surgery [43].

The Spectralis SDOCT is a very useful tool for other macular pathologies, such as retinal vascular occlusive diseases with macular involvement, central serous chorioretinopathy, macular dystrophies, idiopathic perifoveal telangiectasia, and chloroquine retinopathy. There are few studies published in the literature showing its clinical applications. Important advantages with clinical significance of this new technology compared to the time-domain technology are the ability to better visualize the vitreoretinal interface and outer retinal layers, especially the photoreceptors junction, and the possibility to obtain 3-dimensional scans allowing to image structural changes of the vitreoretinal interface and the retina in large areas.

One advantage of the Spectralis OCT is the improved visualization of the choroid. Margolis and Spaide [44] described the measurement of the choroidal thickness using the enhanced depth imaging technique. It is described by positioning the device close enough to the eye to acquire an inverted image within a $5 \times 30$-degree area centered at the fovea and then performing manual measurements from the outer border of the RPE to the inner scleral border. In the normal studied eyes, the subfoveal choroid was thickest and grew thinner at more peripheral measurement points. Also choroidal thickness demonstrated a negative correlation with age. In different reports using the same technique, the author reported enhanced depth imaging of choroidal changes underneath a pigmented epithelial detachment in patients with exudative AMD, thus describing a novel disease entity termed age-related chorioretinal atrophy $[45,46]$. The ability to visualize and quantify choroidal thickness is a very interesting area of research and may be limited to a few SDOCT devices which can overcome the technical limitations of imaging deeper structures such as analogto-digital conversion, wavelength-dependent light scattering, and signal loss in the image path before Fourier transformation.

In conclusion, the combined cSLO/OCT system allows simultaneous recording and interpolation of topographic and tomographic images. Different cSLO imaging modes including infrared and blue reflectance, FAF and fluorescein or ICG angiography can be combined with simultaneous acquisition of OCT. This multimodality combination allows for a better understanding of the pathogenesis of several macular pathologies and improvement of diagnosis and management of patients with macular diseases.

\section{Conflict of Interests}

The authors have no financial interest in any equipment or technique described in the paper.

\section{References}

[1] R. H. Webb, G. W. Hughes, and O. Pomerantzeff, "Flying spot TV ophthalmoscope," Applied Optics, vol. 19, no. 17, pp. 29912997, 1980.

[2] R. H. Webb, G. W. Hughes, and F. C. Delori, "Confocal scanning laser ophthalmoscope," Applied Optics, vol. 26, no. 8, pp. 1492-1499, 1987.

[3] D. Huang, E. A. Swanson, C. P. Lin et al., "Optical coherence tomography," Science, vol. 254, no. 5035, pp. 1178-1181, 1991.

[4] M. R. Hee, J. A. Izatt, E. A. Swanson et al., "Optical coherence tomography of the human retina," Archives of Ophthalmology, vol. 113, no. 3, pp. 325-332, 1995.

[5] M. R. Hee, C. R. Baumal, C. A. Puliafito et al., "Optical coherence tomography of age-related macular degeneration and choroidal neovascularization," Ophthalmology, vol. 103, no. 8, pp. 1260-1270, 1996.

[6] C. A. Puliafito, M. R. Hee, C. P. Lin et al., "Imaging of macular diseases with optical coherence tomography," Ophthalmology, vol. 102, no. 2, pp. 217-229, 1995.

[7] J. S. Schuman, M. R. Hee, A. V. Arya et al., "Optical coherence tomography: a new tool for glaucoma diagnosis," Current Opinion in Ophthalmology, vol. 6, no. 2, pp. 89-95, 1995.

[8] J. S. Schuman, M. R. Hee, C. A. Puliafito et al., "Quantification of nerve fiber layer thickness in normal and glaucomatous eyes using optical coherence tomography: a pilot study," Archives of Ophthalmology, vol. 113, no. 5, pp. 586-596, 1995.

[9] A. Gaudric, B. Haouchine, P. Massin, M. Paques, P. Blain, and A. Erginay, "Macular hole formation: new data provided by optical coherence tomography," Archives of Ophthalmology, vol. 117, no. 6, pp. 744-751, 1999.

[10] D. S. Chauhan, R. J. Antcliff, P. A. Rai, T. H. Williamson, and J. Marshall, "Papillofoveal traction in macular hole formation: the role of optical coherence tomography," Archives of Ophthalmology, vol. 118, no. 1, pp. 32-38, 2000.

[11] P. Massin, C. Allouch, B. Haouchine et al., "Optical coherence tomography of idiopathic macular epiretinal membranes before and after surgery," American Journal of Ophthalmology, vol. 130, no. 6, pp. 732-739, 2000.

[12] P. Massin, B. Haouchine, A. Gaudric et al., "Macular traction detachment and diabetic edema associated with posterior hyaloidal traction ," American Journal of Ophthalmology, vol. 132, no. 4, pp. 599-600, 2001.

[13] P. Massin, A. Girach, A. Erginay, and A. Gaudric, "Optical coherence tomography: a key to the future management of patients with diabetic macular oedema," Acta Ophthalmologica Scandinavica, vol. 84, no. 4, pp. 466-474, 2006.

[14] H. Sánchez-Tocino, A. Alvarez-Vidal, M. J. Maldonado, J. Moreno-Montañés, and A. García-Layana, "Retinal thickness study with optical coherence tomography in patients with 
diabetes," Investigative Ophthalmology and Visual Science, vol. 43, no. 5, pp. 1588-1594, 2002.

[15] R. F. Spaide, D. Wong, Y. Fisher, and M. Goldbaum, "Correlation of vitreous attachment and foveal deformation in early macular hole states," American Journal of Ophthalmology, vol. 133, no. 2, pp. 226-229, 2002.

[16] W. Soliman, B. Sander, and T. M. Jørgensen, "Enhanced optical coherence patterns of diabetic macular oedema and their correlation with the pathophysiology," Acta Ophthalmologica Scandinavica, vol. 85, no. 6, pp. 613-617, 2007.

[17] W. Drexler, U. Morgner, F. X. Kärtner et al., "In vivo ultrahighresolution optical coherence tomography," Optics Letters, vol. 24, no. 17, pp. 1221-1223, 1999.

[18] W. Drexler, U. Morgner, R. K. Ghanta, F. X. Kärtner, J. S. Schuman, and J. G. Fujimoto, "Ultrahigh-resolution ophthalmic optical coherence tomography," Nature Medicine, vol. 7, no. 4, pp. 502-506, 2001.

[19] W. Drexler, H. Sattmann, B. Hermann et al., "Enhanced visualization of macular pathology with the use of ultrahighresolution optical coherence tomography," Archives of Ophthalmology, vol. 121, no. 5, pp. 695-706, 2003.

[20] W. Drexler, U. Morgner, R. K. Ghanta et al., "New technology for ultrahigh resolution optical coherence tomography of the retina," in The Shape of Glaucoma, H. Lemij and J. S. Schuman, Eds., pp. 75-104, Kugler, Berlin, Germany, 2005.

[21] B. Cense, N. A. Nassif, T. C. Chen et al., "Ultrahigh-resolution high-speed retinal imaging using spectral-domain optical coherence tomography," Optics Express, vol. 12, no. 11, pp. 2435-2447, 2004.

[22] W. Drexler, "Ultrahigh-resolution optical coherence tomography," Journal of Biomedical Optics, vol. 9, no. 1, pp. 47-74, 2004.

[23] R. A. Leitgeb, W. Drexler, A. Unterhuber et al., "Ultrahigh resolution fourier domain optical coherence tomography," Optics Express, vol. 12, no. 10, pp. 2156-2165, 2004.

[24] M. Wojtkowski, T. Bajraszewski, I. Gorczynska et al., "Ophthalmic imaging by spectral optical coherence tomography," American Journal of Ophthalmology, vol. 138, no. 3, pp. 412419, 2004.

[25] A. F. Fercher, C. K. Hitzenberger, G. Kamp, and S. Y. El-Zaiat, "Measurement of intraocular distances by backscattering spectral interferometry," Optics Communications, vol. 117, no. 1-2, pp. 43-48, 1995.

[26] G. Hausler and M. W. Lindner, "Coherence radar' and "spectral radar"-new tools for dermatological diagnosis," Journal of Biomedical Optics, vol. 3, no. 1, pp. 21-31, 1998.

[27] E. A. Swanson, J. A. Izatt, M. R. Hee et al., "In vivo retinal imaging by optical coherence tomography," Optics Letters, vol. 18, no. 21, pp. 1864-1869, 1993.

[28] M. Wojtkowski, V. Srinivasan, J. G. Fujimoto et al., "Threedimensional retinal imaging with high-speed ultrahighresolution optical coherence tomography," Ophthalmology, vol. 112, no. 10, pp. 1734-1746, 2005.

[29] H. M. Helb, P. C. Issa, M. Fleckenstein et al., "Clinical evaluation of simultaneous confocal scanning laser ophthalmoscopy imaging combined with high-resolution, spectral-domain optical coherence tomography," Acta Ophthalmologica, vol. 88, no. 8, pp. 842-849, 2010.

[30] U. E. Wolf-Schnurrbusch, V. Enzmann, C. K. Brinkmann, and S. Wolf, "Morphologic changes in patients with geographic atrophy assessed with a novel spectral OCT-SLO combination," Investigative Ophthalmology and Visual Science, vol. 49, no. 7, pp. 3095-3099, 2008.
[31] A. A. Khanifar, A. F. Koreishi, J. A. Izatt, and C. A. Toth, "Drusen ultrastructure imaging with spectral domain optical coherence tomography in age-related macular degeneration," Ophthalmology, vol. 115, no. 11, pp. 1883-1890, 2008.

[32] S. Bearelly, F. Y. Chau, A. Koreishi, S. S. Stinnett, J. A. Izatt, and C. A. Toth, "Spectral domain optical coherence tomography imaging of geographic atrophy margins," Ophthalmology, vol. 116, no. 9, pp. 1762-1769, 2009.

[33] M. Brar, I. Kozak, L. Cheng et al., "Correlation between spectral-domain optical coherence tomography and fundus autofluorescence at the margins of geographic atrophy," American Journal of Ophthalmology, vol. 148, no. 3, pp. 439-444, 2009.

[34] A. Hassenstein and C. H. Meyer, "Clinical use and research applications of Heidelberg retinal angiography and spectraldomain optical coherence tomography-a review," Clinical and Experimental Ophthalmology, vol. 37, no. 1, pp. 130-143, 2009.

[35] C. Cukras, Y. D. Wang, C. B. Meyerle, F. Forooghian, E. Y. Chew, and W. T. Wong, "Optical coherence tomographybased decision making in exudative age-related macular degeneration: comparison of time- vs spectral-domain devices," Eye, vol. 24, no. 5, pp. 775-783, 2010.

[36] A. Giani, D. D. Esmaili, C. Luiselli et al., "Displayed reflectivity of choroidal neovascular membranes by optical coherence tomography correlates with presence of leakage by fluorescein angiography," Retina, vol. 31, no. 5, pp. 942-948, 2011.

[37] S. Yamamoto, T. Yamamoto, M. Hayashi, and S. Takeuchi, "Morphological and functional analyses of diabetic macular edema by optical coherence tomography and multifocal electroretinograms," Graefe's Archive for Clinical and Experimental Ophthalmology, vol. 239, no. 2, pp. 96-101, 2001.

[38] T. Otani, S. Kishi, and Y. Maruyama, "Patterns of diabetic macular edema with optical coherence tomography," American Journal of Ophthalmology, vol. 127, no. 6, pp. 688-693, 1999.

[39] L. Yeung, V. C. Lima, P. Garcia, G. Landa, and R. B. Rosen, "Correlation between spectral domain optical coherence tomography findings and fluorescein angiography patterns in diabetic macular edema," Ophthalmology, vol. 116, no. 6, pp. 1158-1167, 2009.

[40] D. Koleva-Georgieva and N. Sivkova, "Assessment of serous macular detachment in eyes with diabetic macular edema by use of spectral-domain optical coherence tomography," Graefe's Archive for Clinical and Experimental Ophthalmology, vol. 247, no. 11, pp. 1461-1469, 2009.

[41] F. Forooghian, C. Cukras, C. B. Meyerle, E. Y. Chew, and W. T. Wong, "Evaluation of time domain and spectral domain optical coherence tomography in the measurement of diabetic macular edema," Investigative Ophthalmology and Visual Science, vol. 49, no. 10, pp. 4290-4296, 2008.

[42] W. Einbock, L. Berger, U. Wolf-Schnurrbusch, J. Fleischhauer, and S. Wolf, "Predictive factors for visual acuity of patients with diabetic macular edema interpreted form the spectralis HRA+OCT (Heidelberg Engineering)," Investigative Ophthalmology \& Visual Science, vol. 49, Article ID 3473, 2008.

[43] L. K. Chang, H. Koizumi, and R. F. Spaide, "Disruption of the photoreceptor inner segment-outer segment junction in eyes with macular holes," Retina, vol. 28, no. 7, pp. 969-975, 2008.

[44] R. Margolis and R. F. Spaide, "A pilot study of enhanced depth imaging optical coherence tomography of the choroid in normal eyes," American Journal of Ophthalmology, vol. 147, no. 5, pp. 811-815, 2009. 
[45] R. F. Spaide, "Enhanced depth imaging optical coherence tomography of retinal pigment epithelial detachment in age-related macular degeneration," American Journal of Ophthalmology, vol. 147, no. 4, pp. 644-652, 2009.

[46] R. F. Spaide, "Age-related choroidal atrophy," American Journal of Ophthalmology, vol. 147, no. 5, pp. 801-810, 2009. 


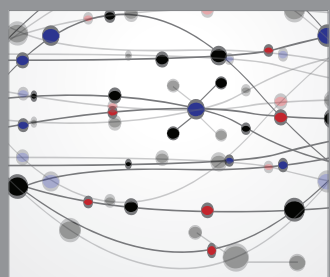

The Scientific World Journal
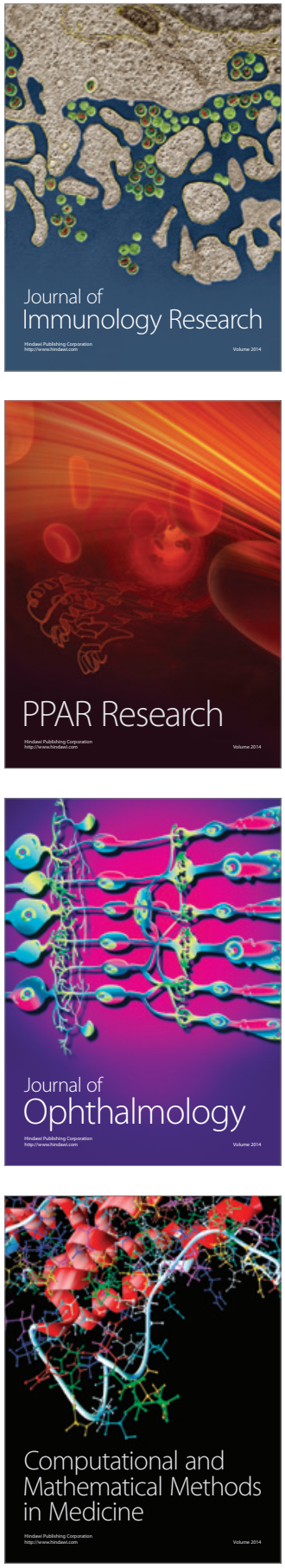

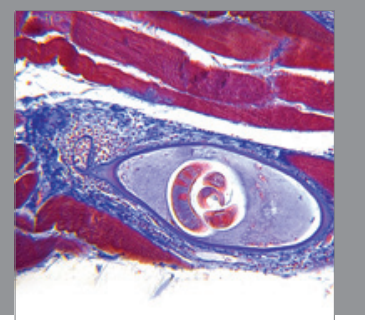

Gastroenterology

Research and Practice
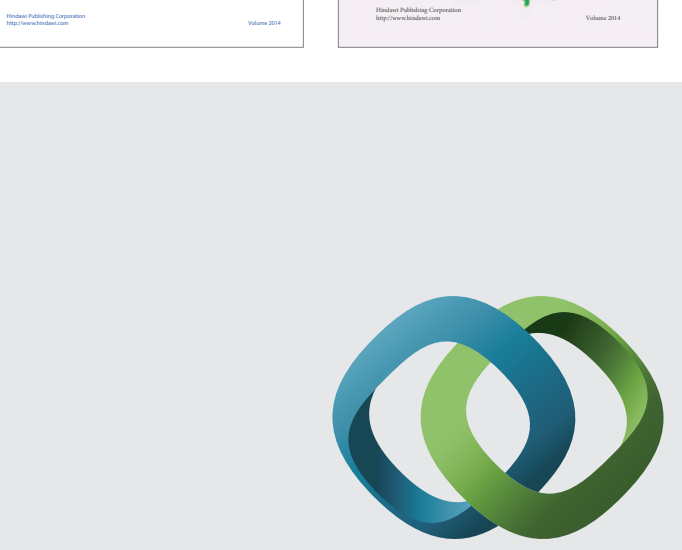

\section{Hindawi}

Submit your manuscripts at

http://www.hindawi.com
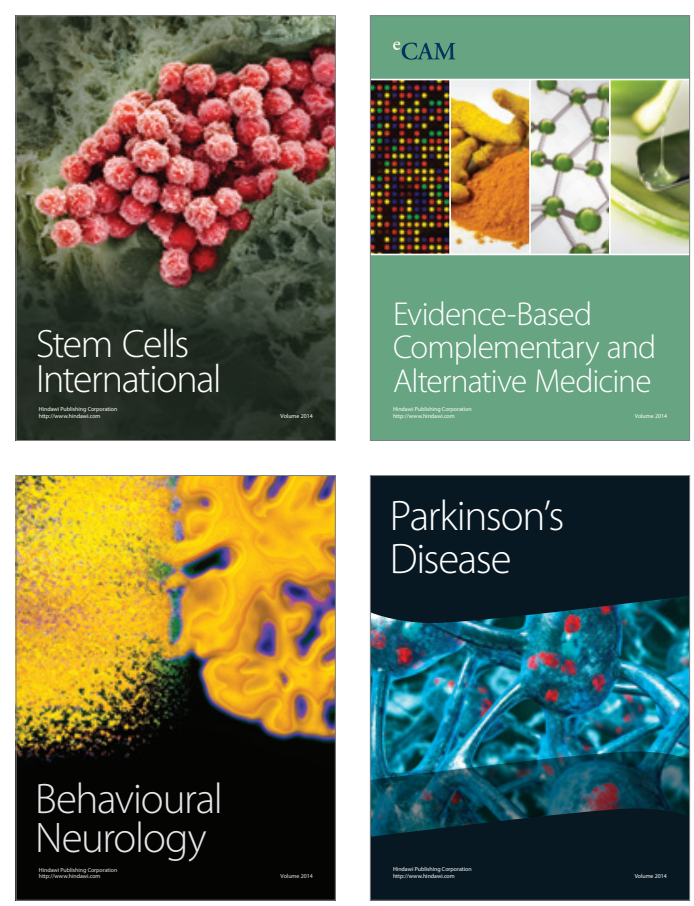

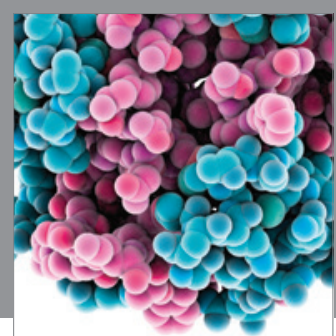

Journal of
Diabetes Research

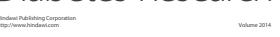

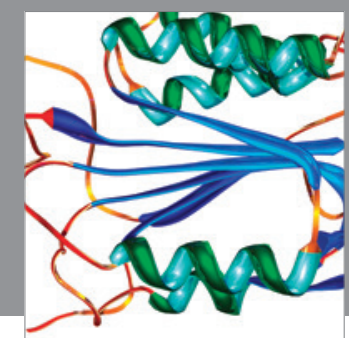

Disease Markers
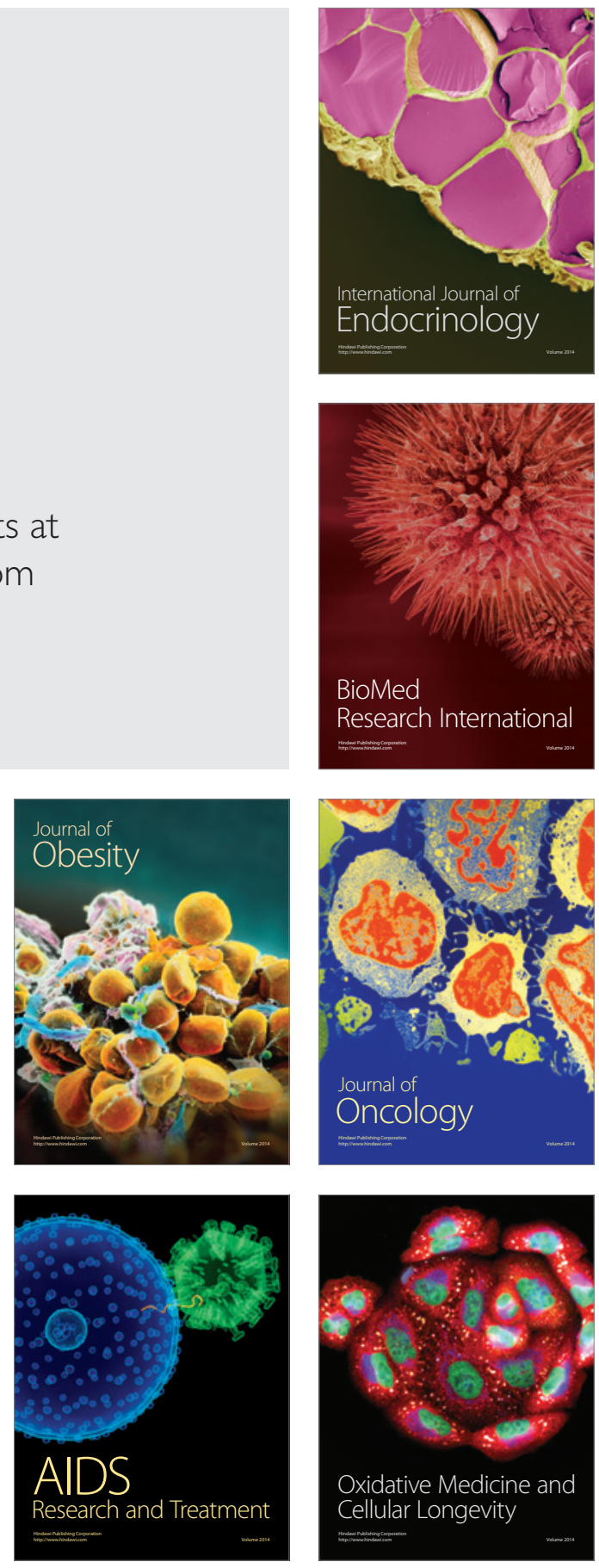